\section{Persistence and}

transmission of recessive deafness and sign language: new insights from village sign languages

European Journal of Human Genetics (2013) 21, 894-896; doi:10.1038/ejhg.2012.292; published online 16 January 2013

The study of the transmission of sign languages can give novel insights into the transmission of spoken languages ${ }^{1}$ and, more generally, into gene-culture coevolution. Over the years, several papers related to the persistence of sign language have been reported. ${ }^{2-6}$ All of these studies have emphasized the role of assortative (non-random) mating by deafness state (ie, a tendency for deaf individuals to partner together) for increasing the frequency of recessive deafness, and hence for the persistence of sign language in a population.

Here, we would like to draw attention to rural signing communities, namely communities where a village sign language ${ }^{7,8}$ is shared among deaf and hearing people, and commonly used in the population. In these communities, assortative mating is unlikely to have been the main driver of increases in frequency of deafness and signing. Consideration of these populations promises to yield more refined and inclusive models for the persistence and transmission of signing. We also present the key elements of such a model.

Aoki and Feldman have previously argued that, in the presence of a single monogenic form of recessive deafness in a population, and under the assumption that no hearing individuals learn to sign, the condition for the persistence of a sign language is

$$
2 l c *[(1-m) q+m]>1
$$

where $l c$ is the fraction of deaf children who learn to sign with only one parent signing, $m$ is the coefficient of assortative mating by deafness state and $q$ is the frequency of the recessive deafness allele in the population of interest.,3 According to this formulation, the persistence of a sign language in a population is ensured by the assortative mating for deaf status and the vertical transmission of the sign language, whereas the contribution of oblique and horizontal transmission to the persistence of a sign language is considered negligible., ${ }^{2,3}$

This model has been recently supported by work on the genetic epidemiology of DFNB1 deafness, ${ }^{4-6}$ highlighting the role of assortative mating in maintaining its high prevalence. The DFNB1 locus is made up of two different genes, GJB2 and GJB6, coding for two gap junction proteins highly expressed in the cochlea (Connexin 26 and 30, respectively). ${ }^{6}$ The close proximity of the GJB2 and GJB6 genes within the genome, and the co-ordinated regulation of their expression in the inner ear, means that DFNB1 deafness can be considered in many respects as a single, monogenic recessive disorder. ${ }^{9,10}$

Nance et $a l^{5}$ compared different US national surveys on deafby-deaf marriages to corroborate the hypothesis that the frequency of DFNB1 deafness among the US population has substantially increased over the last two centuries, ${ }^{4}$ in parallel with the spread of sign language schools. The schooling presumably would have allowed a better integration of deaf people within a broader deaf community, favoring homogamy and higher marriage and reproductive rates, finally increasing genetic fitness. In this way, assortative mating and relaxed selection would have allowed a doubling of the prevalence of the most common form of genetic deafness, DFNB1. ${ }^{5}$

Further support for this hypothesis came through computer simulations of a population of 200000 individuals, with sex ratio 1:1 and a single recessive deafness gene, over 150 generations, with a generation time of 20 years. ${ }^{6}$ Assuming that: (i) the initial fitness of deaf people is 0 (ie, no offspring) and increases up to 1 (ie, normal reproductive rates) in the first five generations, and (ii) assortative mating $(m)$ increases from 0 to 0.9 during the same period, they demonstrated that the increase in the deaf phenotype frequency is greatly accelerated in the presence of assortative mating. These results were further supported by a simulation including three different deafness genes, with only the most common showing an increase in prevalence. As further support for their hypotheses, they cited a higher prevalence of GJB2 deafness in Western Turkey compared with Central and Eastern Turkey, which may reflect a preference in the former region for Western marriage patterns, that is, relatively low rates of consanguinity and high rates of assortative mating among deaf people. ${ }^{4,11}$

Thus, it is clear that assortative mating can have a fundamental role in the persistence of sign language and recessive deafness. Nevertheless, here we would like to emphasize that these simplified models cannot explain many scenarios that are observed in real populations.

We illustrate the key issues by considering the Al-Sayyid Bedouin Sign Language system (ABSL). The Al-Sayyid Bedouins are a highly inbred and endogamous community of around 3500 individuals living in the Negev desert in Israel. ${ }^{12}$ The community was founded about 200 years ago and, since its third generation, consanguineous marriages have become the norm. ${ }^{12,13}$ High endogamy has been favored by rejection of marriages into local neighboring communities, because of cultural isolation. ${ }^{12}$ The genetic isolation of the population, in conjunction with a strong founder effect ${ }^{12,13}$ results in a high prevalence of a profound prelingual neurosensory (non-syndromic) recessive deafness because of mutations in the DFNB1 locus (prevalence $P=2.6-3.3 \%) .{ }^{13-15}$ The Al-Sayyid population is now in its 7 th-8th generation and resides in a single village that is separated from other Bedouin communities. ${ }^{12}$ Birth rates among Bedouins are high, as well as frequency of polygamy, which is reported for $33 \%$ of Bedouin women interviewed in a recent survey (with a mean of 2.3 wives per man in polygamous households). ${ }^{16}$

The cultural isolation of this community has resulted in the birth of a characteristic sign language. Crucially, ABSL shows notable differences from other languages that it may have been in contact with, both sign and spoken, in particular with regard 
to the subject-object-verb and head-modifiers orders. ${ }^{12}$ Moreover, in contrast with other young sign languages, such as Nicaraguan sign language, ABSL is developing in a socially stable community ${ }^{17}$ and is widely used in the population, also by hearing people. ${ }^{14,15,18,19}$

These aspects have led linguists to recognize ABSL as a new independent developing sign language. ${ }^{1,17}$ The first deaf people appeared in the Al-Sayyid population three generations ago, ${ }^{17}$ yielding an estimate of the language age at around 70-80 years. ABSL is increasingly drawing attention as it may additionally provide insights into the origins and transmission of spoken language. ${ }^{1}$

Anthropological reports provide several details on Al-Sayyid sociolinguistic aspects. ${ }^{14,15,18,19}$ Deaf individuals are fully integrated in the community, with a normal marriage rate and hence there is no selective pressure against deafness. Given the cultural habits of Bedouins, which include widespread polygamy and extended family households, many children are exposed to ABSL very early in their childhood, regardless of hearing status. This is because the very high prevalence of deafness and the mating patterns make it likely that in every family there is at least one deaf subject. The recessive transmission of deafness, jointly with the pattern of consanguineous/ endogamous marriages, may have contributed to this situation as well. ${ }^{20}$ Also, the relatively high birth rates might affect early language acquisition, as they result in more older signing siblings, more carriers and more deaf family members in overlapping generations. All of these anthropological factors have surely contributed to the spread of ABSL also to many hearing individuals.

We assessed the validity of the previous predictions made on the persistence of sign language in a population, ${ }^{2,3}$ when applied to a system such as ABSL. To do so we used available information from previous genetic, anthropological and linguistic reports to infer ABSL realistic estimates for the parameters contained in (1a). Considering that: (i) in a small, stable isolated community where a sign language is widely used, every deaf individual will learn it, that is, $1 \mathrm{c} \sim 1$; (ii) a conservative estimate of the prevalence is $P=0.033$ (entailing $q=\sqrt{ } 0.033 \approx 0.18$ ); and (iii) in the ABSL community almost every deaf individual gets married with a hearing one, ${ }^{14,15}$ that is, $m \sim 0$; (1a) will become

$$
2 * 1 *[(1-0) * 0.18+0]=0.36<1
$$

From (1b) it is clear that the model by Aoki and Feldman does not hold under the assumption that no hearing individuals learn to sign. However, although Aoki and Feldman did not allow for a high probability that hearing children will learn to sign, the model can also be tested under this hypothesis. In this case, for the persistence of sign language it has to be

$$
\lambda_{\max }>1
$$

Where $\lambda_{\max }$ is a characteristic index included in the range

$$
2 l b<\lambda_{\max }<2 l c
$$

and where $l c$ and $l b$ are the fractions of deaf and hearing children (respectively) who learn to sign with only one parent signing (see original article for more details). ${ }^{2}$ In other words, at least half of the individuals in the population with one parent signing should learn to sign or, alternatively, the only signing parent must be able to transmit the sign language with greater than one-half the efficiency of two signing parents. ${ }^{2}$

Although we can roughly estimate $l c \sim 1$, a current lack of empirical data on the $\mathrm{lb}$ parameter does not allow a full verification of the Aoki and Feldman model in the ABSL system and it would be worth collecting data for this purpose.
The peculiarity of the ABSL system also led us to test this population against the hypotheses on genetic epidemiology of recessive deafness proposed by Nance et al. ${ }^{4-6}$ The ABSL scenario is clearly inconsistent with a major role of assortative mating. Among Al-Sayyid Bedouins the joint effect of high consanguinity, endogamy and random mating make it possible to reach increasing values of prevalence of DFNB1 deafness in the community: from $2.6 \%$ in 1995 to $3.3 \%$ in 2004 . $^{13-15}$

Although Nance et $a l^{5}$ noted the role of consanguinity/endogamy (along with genetic drift) in the initial survival and expression of new recessive mutations in small communities, in their analysis this role is restricted to the early spread of deafness in the community, ${ }^{5}$ as confirmed by the fact that in their simulations $m$ increases from 0 to $90 \%$ in only 5 generations. ${ }^{6}$ This is not in line with what is happening in the ABSL community, where $m \approx 0 .{ }^{14,15}$ Nance et al noted the potential importance of linguistic homogamy, rather than assortative mating by deafness per se, in the marital patterns of deaf populations. ${ }^{4}$ In other words, it may be the ability to sign, rather than the deaf status itself, which is the real factor determining high assortative mating rates among deaf people. However, although deafness and signing coincide in Western communities, this co-segregation is typically not observed in village communities like Al-Sayyid Bedouins. Perhaps consanguineous marriages may be considered as a kind of nonrandom mating, based on genotype instead of the hearing/deaf status. Nevertheless, the effects of assortative and consanguineous matings are easily distinguishable. Not only does assortative mating generate gametic phase disequilibrium for deafness genes, that is, the nonrandom association and gametic transmission of alleles at unlinked loci that have similar effects on the phenotype, ${ }^{6}$ but it is also expected to generate less homozygosity than consanguineous mating at a genomewide level. ${ }^{21}$

Furthermore, in a highly endogamous and young community like Al-Sayyid Bedouins, the average inbreeding coefficient $(F)$ is relatively high. The probability of marrying a DFNB1 mutation carrier is notably high in this 'random inbreeding' situation, where, whatever the partner choice of an individual, it is highly likely that he/she would marry a related subject. ${ }^{22}$ This underscores an additional difference between assortative and consanguineous mating: whereas the former implies the nonrandom assortment of deafness alleles in the allelic pool of a population, leading to an increase in the prevalence more than in the allelic frequency, under random consanguineous mating $q$ actually increases and so does $P$. In other words, under assortative mating there is no panmixy (ie, no random mating) in the population and $q \rightarrow P$ (instead of $P=q^{2}$ as for Hardy-Weinberg Equilibrium) whereas under random inbreeding $P \approx q^{2}$, as this condition can be better assimilated to a panmictic population.

It may be argued that the computer simulations run by Nance and Kearsey ${ }^{6}$ assume a population size much larger than the current AlSayyid Bedouins. However, the authors reach the same conclusions on populations with size and characteristics similar to the ABSL system, namely the Bengkala community in Northern Bali. ${ }^{4}$ In this community, a sign language (Kata Kolok) has emerged and evolved also in the context of a highly endogamous village with high incidence of recessive deafness ${ }^{23}$ because of a mutation in the MYO15A gene. ${ }^{24,25}$ Thus, there is a real risk of conclusions becoming overgeneralized to many or all possible cultural, demographic and genetic scenarios.

To sum up, the predictions made by Aoki and Feldman can apply only in part to the ABSL system, and the model should be tested on 
empirical data collected from the ABSL community for this purpose. Similarly, also the theories proposed by Nance et $a l^{5}$ should be further tested for their general applicability.

In our view, existing models for the persistence of sign language, and previous theories on the genetic epidemiology of recessive deafness, have been based on genetic and linguistic scenarios that are typical of Western populations (which are presumably the target they were designed for). However, the existence of rural signing communities, as in the case of ABSL, presents unexpected characteristics that do not fit well with models based on Western populations. Indeed, endogamy/inbreeding and cultural integration of deaf people in the community seem to be the fundamental factors for the birth and persistence of village sign languages.

Senghas ${ }^{1}$ has posited that a clustering of a critical mass of signers, in the specific cultural context and communicative networks of a community, can ensure the persistence and transmission of sign languages even when there is only a low overall frequency of signers. This 'critical mass' hypothesis could be the unifying concept to explain both the persistence of recessive deafness and of sign languages in very small, inbred populations with 'non-Western' cultural habits and marital patterns.

We propose that a more general model should include the critical mass estimate. For the ABSL case, this could be inferred from: (i) the allelic frequency of the DFNB1 deafness in the Al-Sayyid population, which could be computed relying on the average $F$, the DFNB1 mutation spectrum and genotype distribution of a small sample of deaf subjects without recurring to classical descriptive epidemiology studies; ${ }^{26}$ (ii) a general $F$ estimate, inferred through pedigree/ genomic/consanguineous marriages frequency data; (iii) the effective population size $\left(N_{\mathrm{e}}\right)$; (iv) the assortative mating coefficient $(m)$ of the population of interest; (v) 'polygamy coefficients' (ie, number of wives per reproductively active man) accounting for both possible effects on the increase in the allelic frequency of deafness and, most of all, for the spread of the exposure to the sign language in the population; (vi) a vertical transmission parameter (with a coefficient that can explain the variability in the intensity of vertical transmission among the different kinds of marriages, namely deaf-deaf, hearingdeaf and hearing-hearing); and (vii) horizontal and oblique transmission parameters. Moreover, the model should consider the contribution of other potentially important cultural elements to the persistence of a sign language, such as the age of acquisition of the sign language and the social pressures and opportunities leading to its learning and active usage. ${ }^{1}$ Therefore, in addition to a quantitative estimation of the 'critical mass of signers', it will be useful to qualitatively refine it taking into account the larger sociolinguistic context.

Such a general model for the persistence of sign languages requires several basic assumptions. For example, it would assume the quasiisolation of the system, from the point of view of genetics (negligible gene flow into/out of the population) as well as linguistics (reduced influence from other spoken and signed languages and from the potential disruptive effects of cochlear implants). Crucially, it will have a broader significance beyond the emergence, evolution and maintenance of deaf village signs, suggesting general processes affecting the evolution of our capacity for language, as well as the language's own capacity to adapt to pressures emerging from its speakers' biology. 27

\section{CONFLICT OF INTEREST}

The authors declare no conflict of interest.

\author{
Alessandro Gialluisi ${ }^{1}$, Dan Dediu ${ }^{1,2}$, Clyde Francks ${ }^{1,2}$ and \\ Simon E Fisher ${ }^{1,2}$ \\ ${ }^{1}$ Language and Genetics Department, Max Planck Institute for \\ Psycholinguistics, Nijmegen, The Netherlands; \\ ${ }^{2}$ Donders Institute for Brain, Cognition and Behaviour, Radboud \\ University Nijmegen, Nijmegen, The Netherlands \\ E-mail:simon.fisher@mpi.nl
}

1 Senghas A: Language emergence: clues from a new Bedouin sign language. Curr Biol 2005; 15: 463-465.

2 Aoki K, Feldman MW: Recessive hereditary deafness, assortative mating and grandparental transmission facilitate the persistence of a sign language persistence of a sign language. Theor Popul Biol 1991; 39: 358-372.

3 Feldman MW, Aoki K: Assortative mating and persistence of a sign language. Theor Popul Biol 1992; 42: 107-116.

4 Arnos KS, Welch KO, Tekin M et al: A comparative analysis of the genetic epidemiology of deafness in the United States in two sets of pedigrees collected more than a century apart. Am J Hum Genet 2008; 83: 200-207.

5 Nance WE, Liu XZ, Pandya A: Relation between choice of partner and high frequency of connexin-26 deafness. Lancet 2000; 356: 500-501.

6 Nance WE, Kearsey MJ: Relevance of connexin deafness (DFNB1) to human evolution. Am J Hum Genet 2004; 74: 1081-1087.

7 Meir I, Sandler W, Padden C, Aronoff M: Emerging sign languages; in Marschark M, Spencer P (eds) Oxford Handbook of Deaf Studies, Language, and Education. Oxford: Oxford University Press, 2010; Vol 2, pp 267-280.

8 Zeshan U: Village sign languages-a commentary; in: Napoli. Donna, Jo, Mathur Gaurav (eds) Deaf Around the World: the Impact of Language. Oxford: Oxford University Press, 2010; pp 221-230.

9 del Castillo I, Villamar M, Moreno-Pelayo MA et al: A deletion involving the connexin 30 gene in nonsyndromic hearing impairment. N Engl J Med 2002; 346 243-249.

10 Ortolano S, Di Pasquale G, Crispino G, Anselmi F, Mammano F, Chiorini JA Coordinated control of connexin 26 and connexin 30 at the regulatory and functional level in the inner ear. Proc Natl Acad Sci USA 2008; 105 $18776-18781$.

11 Tekin M, Arici ZS: Genetic epidemiological studies of congenital/prelingual deafness in Turkey: population structure and mating type are major determinants of mutation identification. Am J Med Genet A 2007; 143: 1583-1591.

12 Sandler W, Meier I, Padden C, Aronoff M: The emergence of grammar: systematic structure in a new language. Proc Natl Acad Sci USA 2005; 102: 2661-2665.

13 Scott DA, Carmi R, Elbedour K, Duyk GM, Stone EM, Sheffield VC: Nonsyndromic autosomal recessive deafness is linked to the DFNB1 locus in a large inbred Bedouin family from Israel. Am J Hum Genet 1995; 57: 965-968.

14 Kusters A: Deaf utopias? Reviewing the sociocultural literature on the world's 'Martha's Vineyard Situations'. J Deaf Stud Deaf Educ 2010; 15: 3-16.

15 Kisch S: Negotiating (genetic) deafness in a Bedouin community; in Van Cleve JV (ed) Genetics, Disability, and Deafness. Washington: Gallaudet University Press, 2004; pp 195-227.

16 Al-Krenawi A, Graham JR: A comparison of family functioning, life and marital satisfaction, and mental health of women in polygamous and monogamous marriages. Int J Soc Psychiatry 2006; 52: 5-17.

17 Goldin-Meadow S: Watching language grow. Proc Natl Acad Sci USA 2005; 102: 2271-2272.

18 Kisch S: Disablement, gender and deafhood among the Negev Arab-Bedouin. Disability Studies Quarterly 2007; 27, from http://www.dsq-sds.org/article/view/45/45.

19 Kisch S: 'Deaf discourse': the social construction of deafness in a Bedouin community in the Negev. Med Anthropol 2010; 27: 283-313.

20 Lane H, Pillard RC, French M: Origins of the American deaf-world: assimilating and differentiating societies and their relation to genetic patterning. Sign Language Studies 2000; 1: 17-44.

21 Crow J, Kimura M: An Introduction to Population Genetics Theory. New York: Harpe and Row, 1970

22 Cavalli Sforza LL, Moroni A, Zei G: Consanguinity, Inbreeding and Genetic Drift in Italy. Princeton, NJ: Princeton University Press, 2004.

23 De Vos C: Sign-Spatiality in Kata Kolok: How A Village Sign Language of Bali Inscribes its Signing Space, PhD DissertationNijmegen: Max Planck Institute for Psycholinguistics, 2012.

24 Friedman TB, Liang $\mathrm{Y}$, Weber $\mathrm{JL}$ et al: A gene for congenital, recessive deafness DFNB3 maps to the pericentromeric region of chromosome 17. Nat Genet 1995; 9: 86-91.

25 Wang A, Liang Y, Fridell RA et al: Association of unconventional myosin MYO15 mutations with human nonsyndromic deafness DFNB3. Science 1998; 280: 1447-1451.

26 Gialluisi A, Pippucci T, Anikster Y et al: Estimating the allele frequency of autosomal recessive disorders through mutational records and consanguinity: the homozygosity index (HI). Ann Hum Genet 2012; 76: 159-167.

27 Dediu D: Are languages really independent from genes? If not, what would a genetic bias affecting language diversity look like? Hum Biol 2011; 83: 279-296. 